07

\title{
Наногетероструктуры с квантовыми \\ точками CdTe/ZnMgSeTe \\ для однофотонных источников, формируемые методом \\ молекулярно-пучковой эпитаксии
}

\author{
(C) С.В. Сорокин, И.В. Седова, К.Г. Беляев, М.В. Рахлин, \\ М.А. Яговкина, А.А. Торопов, С.В. Иванов
}

Физико-технический институт им. А.Ф. Иоффре РАН, Санкт-Петербург,

Россия

E-mail: sorokin@beam.ioffe.ru

Поступило в Редакцию 12 октября 2017 г.

Представлены результаты исследования технологии выращивания методом молекулярно-пучковой эпитаксии на подложках $\operatorname{InAs}(001)$, конструкции и люминесцентных свойств гетероструктур с квантовыми точками (КТ) $\mathrm{CdTe} / \mathrm{Zn}(\mathrm{Mg})(\mathrm{Se}) \mathrm{Te}$. Приведены результаты рентгеноструктурных исследований короткопериодных сверхрешеток $\mathrm{ZnTe} / \mathrm{MgTe} / \mathrm{MgSe}$, используемых в качестве широкозонных барьеров в структурах с КТ CdTe/ZnTe для эффективного ограничения дырок. Показано, что при конструировании данных сверхрешеток необходимо учитывать замещение атомов Те селеном на гетероинтерфейсах $\mathrm{MgSe} / \mathrm{ZnTe}$ и $\mathrm{MgTe} / \mathrm{MgSe}$ В гетероструктурах с KT $\mathrm{CdTe} / \mathrm{Zn}(\mathrm{Mg})(\mathrm{Se}) \mathrm{Te}$ продемонстрирована фотолюминесценция КТ вплоть до $300 \mathrm{~K}$. Спектры микрофотолюминесценции при $T=10 \mathrm{~K}$ демонстрируют набор линий от одиночных КТ $\mathrm{CdTe} / \mathrm{ZnTe}$, при этом поверхностная плотность КТ может быть оценена как $\sim 10^{10} \mathrm{~cm}^{-2}$.

DOI: $10.21883 /$ PJTF.2018.06.45772.17075

В последние два десятилетия прилагаются значительные усилия в области разработки источников однофотонного излучения. Высокоэффективный однофотонный источник потенциально имеет множество важных приложений. В частности, он востребован в квантовых вычислениях и в телекоммуникационных системах, поскольку обеспечивает абсолютную безопасность обмена данными с использованием прото- 
колов квантовой криптографии. В качестве реализации излучателей одиночных фотонов весьма перспективным является подход, связанный с использованием полупроводниковых квантовых точек (КТ). Эмиссия одиночных фотонов в эпитаксиальных структурах с КТ была продемонстрирована для различных систем соединений $\mathrm{A}^{3} \mathrm{~B}^{5}$ и $\mathrm{A}^{2} \mathrm{~B}^{6}$, в том числе для $\mathrm{KT} \mathrm{CdSe} / \mathrm{Zn}(\mathrm{S}, \mathrm{Se})[1,2]$ и $\mathrm{CdTe} / \mathrm{ZnTe}[3,4]$.

Одним из критических параметров, способствующих наблюдению люминесценции одиночных КТ, является их низкая поверхностная плотность. Для формирования КT CdTe/ZnTe с низкой поверхностной плотностью методом молекулярно-пучковой эпитаксии (МПЭ) наиболее апробированным является использование режима так называемой термической активации (ТА) КТ, при котором формирование КТ индуцируется уменьшением поверхностной энергии напряженного двумерного (2D) слоя CdTe при покрытии его слоем аморфного Те с последующей быстрой десорбцией Те-покрытия [5]. Влияние различных параметров (номинальной толщины слоя CdTe KT, температуры осаждения и температуры отжига KT CdTe) при использовании режима ТA на фотолюминесцентные свойства и плотность формируемых KT CdTe/ZnTe подробно исследовано в работах [5-8], где было показано, что поверхностная плотность КТ может достигать значения $\sim 10^{10} \mathrm{~cm}^{-2}[5,8]$ и менее [6]. В [4] на меза-структурах шириной $200 \mathrm{~nm}$, изготовленных на основе структур с KT CdTe/ZnTe, сформированных в режиме ТА, удалось наблюдать эффект антигруппировки фотонов со средним значением корреляционной функции $g^{(2)}(0)$, равным $0.16( \pm 0.04)$ при $T=8 \mathrm{~K}$, что указывает на однофотонную природу излучения КТ. Однако увеличение рабочей температуры однофотонных источников на основе КТ $\mathrm{CdTe} / \mathrm{ZnTe}$ требует использования широкозонных барьеров $\mathrm{ZnMgSeTe}$, которые предназначены для улучшения ограничения дырок за счет увеличения разрыва валентной зоны на гетерогранице $\mathrm{CdTe} / \mathrm{ZnMgSeTe}$ по сравнению со случаем CdTe/ZnTe.

В настоящей работе сообщается о выращивании методом МПЭ наноструктур с KT CdTe, внедренными в барьеры квазичетверного твердого раствора ZnMgSeTe, реализованного в виде короткопериодных сверхрешеток (CP) $\mathrm{ZnTe} / \mathrm{MgTe} / \mathrm{MgSe}$ (период CP $T_{\mathrm{SL}} \sim 2 \mathrm{~nm}$ ), и исследовании их структурных и фотолюминесцентных свойств. Структуры продемонстрировали эффективную фотолюминесценцию (ФЛ) КТ вплоть до комнатной температуры, а также спектрально разрешенные линии излучения из одиночных КТ при $T=10 \mathrm{~K}$.

Письма в ЖТФ, 2018, том 44, вып. 6 
Структуры с КТ CdTe/Zn $(\mathrm{Mg})(\mathrm{Se}) \mathrm{Te}$ были выращены псевдоморфно на подложках $\operatorname{InAs}(001)$ с предварительно осажденным на них буферным слоем - $0.2 \mu \mathrm{m}$ InAs - при использовании двухкамерной установки МПЭ (SemiTEq, Россия) при температуре подложки $T_{S}=295-300^{\circ} \mathrm{C}$. Стандартные эффузионные источники $\mathrm{Zn}, \mathrm{Cd}, \mathrm{Mg}$, $\mathrm{Te}$, а также клапанный источник Se были использованы в качестве источников молекулярных пучков. Нагрев подложки осуществлялся радиационно-бесконтактным способом. Рост был инициирован одновременным открытием заслонок $\mathrm{Zn}$ и Те на поверхность буферного слоя InAs c реконструкцией $(2 \times 4)$ As [9]. Рост КТ CdTe контролировался in situ методом дифракции быстрых электронов на отражение (ДБЭО). Структуры с КТ $\mathrm{CdTe} / \mathrm{Zn}(\mathrm{Mg})(\mathrm{Se}) \mathrm{Te}$ состояли из буферного слоя $\mathrm{ZnTe}$ толщиной 5 моноатомных слоев (ML), короткопериодной CP $\mathrm{ZnTe} / \mathrm{MgTe} / \mathrm{MgSe}$ толщиной $\sim 150 \mathrm{~nm}$, слоя KT CdTe (при номинальной толщине CdTe $\sim 3 \mathrm{ML}$ ), отделенного с обеих сторон от слоев СР спейсерами $\mathrm{ZnTe}$ толщиной $3 \mathrm{ML}$, и верхней $\mathrm{CP} \mathrm{ZnTe} / \mathrm{MgTe} / \mathrm{MgSe}$ толщиной $\sim 45 \mathrm{~nm}$. КТ CdTe/ZnTe формировались в режиме TA, при этом увеличение $T_{S}$ после десорбции слоя аморфного теллура проводилось в отсутствие потока Те на поверхность роста [8]. Дополнительно также были выращены структуры, содержащие „чистые“ $\mathrm{CP} \mathrm{ZnTe} / \mathrm{MgTe} / \mathrm{MgSe}$. Для анализа структурных свойств образцов были измерены $\theta-2 \theta$-кривые рентгеновской дифракции (РД) вблизи рефлекca $\operatorname{InAs}(004)$ с использованием высокоразрешающего рентгеновского дифрактометра D8 Discover (Bruker, Германия), а также проведены измерения методом просвечивающей электронной микроскопии (ПЭМ) в геометрии поперечного сечения (микроскоп Philips EM-420). Оптические свойства структур с КТ исследовались методом спектроскопии фотолюминесценции, а также низкотемпературной микро-ФЛ. Спектры регистрировались с помощью охлаждаемой CCD-камеры, в качестве источников возбуждения использовались лазерные линии с $\lambda=377$ и $404 \mathrm{~nm}$ (CUBE-лазер, Coherent).

Идея заменить четверной твердый раствор $\mathrm{ZnMgSeTe}$ короткопериодными CP типа ZnTe/MgTe/MgSe была предложена и реализована ранее авторами [10] при МПЭ высокоотражающих широкополосных распределенных брэгговских отражателей, согласованных по периоду решетки c ZnTe. В случае применения СР существенно упрощается управление составом растущего слоя, которое реализуется посредством выбора соответствующих толщин бинарных соединений, об- 
разующих СР. В настоящей работе все слои короткопериодных СР $\mathrm{ZnTe} / \mathrm{MgTe} / \mathrm{MgSe}$ с периодом $\sim 2 \mathrm{~nm}$ были выращены в режиме МПЭ в условиях обогащения поверхности элементами VI группы. Приоритет формирования интерфейсных слоев в СР определяется различием в энтальпии образования соответствующих бинарных соединений (для решетки цинковой обманки - ZB)

$$
\Delta H_{298}^{0}(\mathrm{MgSe})>\Delta H_{298}^{0}(\mathrm{ZnSe})>\Delta H_{298}^{0}(\mathrm{MgTe})>\Delta H_{298}^{0}(\mathrm{ZnTe})(\mathrm{ZB}) .
$$

Таким образом, исходя из условия (1) следует ожидать формирования слоя $\mathrm{ZnSe}$ на интерфейсе $\mathrm{MgSe} / \mathrm{ZnTe}$, в то время как частичное замещение атомов теллура селеном на интерфейсе $\mathrm{MgTe} / \mathrm{MgSe}$ должно приводить к увеличению доли $\mathrm{MgSe}$ в СР. Эти предположения подтверждаются моделированием $\theta-2 \theta$-кривых РД двух структур с $\mathrm{CP} \mathrm{ZnTe} / \mathrm{MgTe} / \mathrm{MgSe}$, измеренных вблизи рефлекса InAs(004) (рис. 1). Хорошее согласие экспериментальных и модельных кривых РД достигается в предположении формирования слоев $\mathrm{ZnSe}$ толщиной $\sim 1.1 \mathrm{ML}$ на границах раздела $\mathrm{MgSe} / \mathrm{ZnTe}$ за счет соответствующего уменьшения толщины слоев ZnTe, а также увеличения толщины слоев MgSe на 0.6-0.8 ML на интерфейсах $\mathrm{MgTe} / \mathrm{MgSe}$ за счет соответствующего уменьшения толщины прилежащих слоев $\mathrm{MgTe}$. Помимо заданного периода СР при моделировании кривых РД также использовались данные по скоростям роста бинарных соединений, составляющих $\mathrm{CP}$, определенных по осцилляциям интенсивности отраженного пятна в ДБЭО. Дополнительно также учитывалось различие между скоростями роста слоев $\mathrm{MgTe}$ и $\mathrm{MgSe}$, выращиваемых при постоянном потоке $\mathrm{Mg}$, за счет различия периода решетки соответствующих соединений.

Формирование $\mathrm{ZnSe}-$ содержащих слоев толщиной 1.5 и $2.5 \mathrm{ML}$ на интерфейсах $\mathrm{ZnTe} / \mathrm{CdSe}$ при МПЭ СР типа $\mathrm{ZnTe} / \mathrm{CdSe}$ ранее было также отмечено в работе [11]. Однако в отличие от настоящей работы слои $\mathrm{ZnTe}$ в [11] были выращены в Zn-обогащенных условиях. В любом случае, несмотря на то что детали микроструктуры интерфейсов в $\mathrm{CP} \mathrm{ZnTe} / \mathrm{MgTe} / \mathrm{MgSe}$ неизвестны, их вклад необходимо учитывать при конструировании СР, изорешеточных с подложкой InAs.

Изображение структуры с КТ CdTe/ZnTe в барьерах CP $\mathrm{ZnTe} / \mathrm{MgTe} / \mathrm{MgSe}$, полученное методом ПЭМ в геометрии поперечного сечения, представлено на рис. 2. На изображении четко различаются как слои СР, так и слой KT CdTe/ZnTe. Период CP составляет $\sim 2.2 \mathrm{~nm}$ и слегка отличается для верхней и нижней $\mathrm{CP}$, что может быть вызвано

7 Письма в ЖТФ, 2018, том 44, вып. 6 

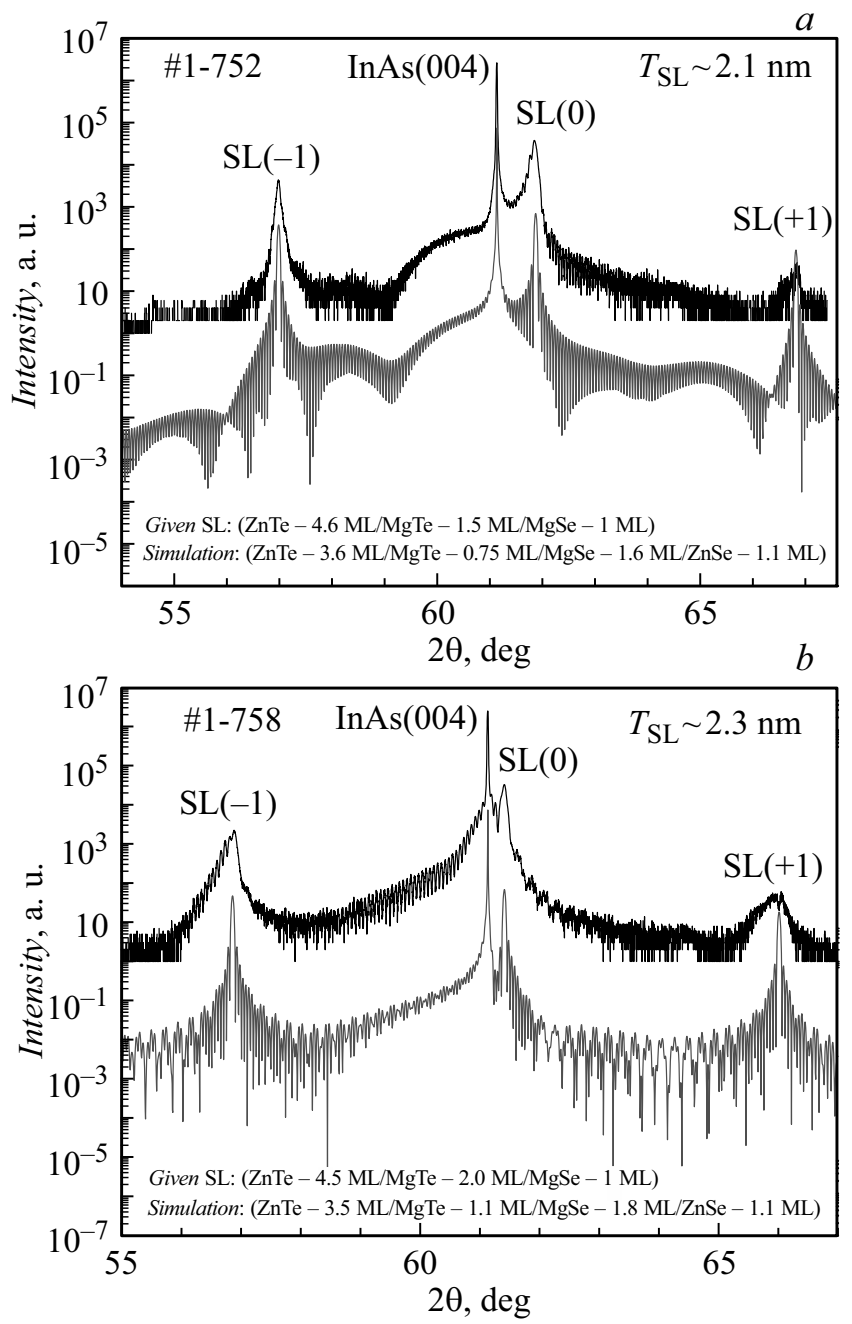

Рис. 1. Кривые РД вблизи рефлекса $\operatorname{InAs}(004)$ для структуры с CP $\mathrm{ZnTe} / \mathrm{MgTe} / \mathrm{MgSe}$ толщиной $0.2 \mu \mathrm{m}(a)$ и для структуры с KT CdTe/ZnTe, ограниченными барьерами в виде короткопериодных CP (SL) ZnTe/MgTe/MgSe (b). Верхняя кривая — эксперимент, нижняя - результат моделирования. 


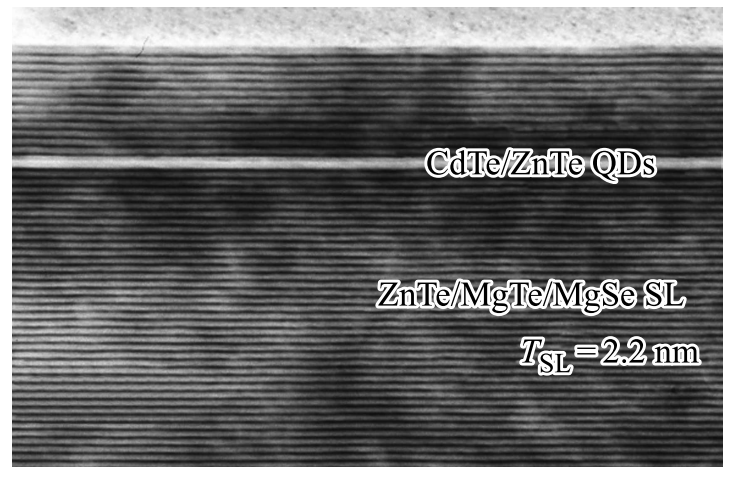

$40 \mathrm{~nm}$

Рис. 2. ПЭМ-изображение в геометрии поперечного сечения структуры c KT CdTe/ZnTe, ограниченными барьерами в виде короткопериодных СР $\mathrm{ZnTe} / \mathrm{MgTe} / \mathrm{MgSe}$. Период СР составляет $\sim T_{\mathrm{SL}}=2.2 \mathrm{~nm}$.

вариацией потоков основных элементов из-за длительного прерывания роста, требуемого для снижения $T_{S}$ (до $1.5-2 \mathrm{~h}$ в зависимости от типа используемой установки МПЭ) до температуры осаждения слоя аморфного теллура $\left(<50^{\circ} \mathrm{C}\right)$ при формировании КТ CdTe/ZnTe в режиме ТА. Более того, длительная остановка роста может приводить к образованию заметной плотности дефектов упаковки на гетероинтерфейсе KT/ZnTe [12]. Вместе с тем ПЭМ-изображения демонстрируют полную когерентность всей структуры с InAs.

Спектры ФЛ структуры с КТ $\mathrm{CdTe} / \mathrm{Zn}(\mathrm{Mg})(\mathrm{Se}) \mathrm{Te}$ (рис. 3) демонстрируют пики, связанные со СР и КТ CdTe/ZnTe, причем пик от СР доминирует при низких температурах. Отчетливо наблюдается сложная структура пика ФЛ от КТ, который начинает преобладать в спектре при $T>50 \mathrm{~K}$. Более узкий коротковолновый пик $\left(E_{\max } \sim 2.2 \mathrm{eV}\right)$ может быть связан с излучением из так называемого смачивающего слоя, в то время как широкий длинноволновый пик $\left(E_{\max } \sim 1.9-2.0 \mathrm{eV}\right)$ связан с излучением непосредственно из КТ. За счет большего ограничения дырок ФЛ КТ сохраняется вплоть до комнатной температуры, при этом интенсивность пика ФЛ КТ при изменении температуры от $T=8$ до $150 \mathrm{~K}$ падает всего лишь в 4-5 раз (по сравнению с 25-кратным снижением в случае KT CdTe, сформированных в барьерах бинарного ZnTe).

7* Письма в ЖТФ, 2018, том 44, вып. 6 


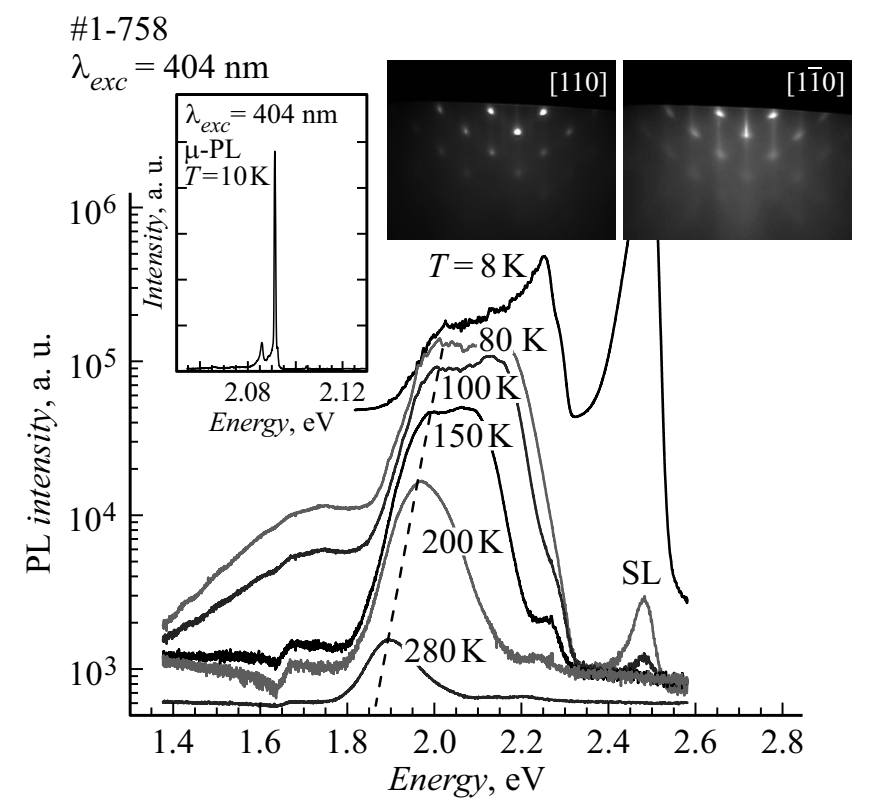

Рис. 3. Спектры ФЛ $(T=8-280 \mathrm{~K})$ структуры с КТ CdTe/ZnTe, ограниченными барьерами в виде короткопериодных CP ZnTe/MgTe/MgSe. На вставках изображения ДБЭО в двух ортогональных направлениях [110] и [110] при формировании КТ (справа) и спектр низкотемпературной микро-ФЛ при возбуждении через пинхол $(T=10 \mathrm{~K})$ (слева).

На процесс формирования КТ CdTe/ZnTe сильное влияние оказывает остаточный фон Se в камере МПЭ $[4,13]$. Наличие заметного фона $\mathrm{Se}$ в ростовой камере приводит к неконтролируемому замещению теллура селеном на поверхности слоя CdTe, что вызывает как изменение энергии поверхности, так и уменьшение упругой энергии и препятствует TA-формированию КТ [4]. Радикальное снижение потокда селена при МПЭ слоев $\mathrm{MgSe}$ при выращивании $\mathrm{CP} \mathrm{ZnTe} / \mathrm{MgTe} / \mathrm{MgSe}$ позволило оптимизировать процесс формирования КТ и существенно сократить время паузы при закрытом клапанном источнике Se, требуемой для снижения фона $\mathrm{Se}$ в ростовой камере, при последующем формировании КТ CdTe в режиме ТА. При этом в ДБЭО наблюдался отчетливый 2D-3D-переход морфологии поверхности (вставки справа на рис. 3),

Письма в ЖТФ, 2018, том 44, вып. 6 
как и случае МПЭ КТ CdTe/ZnTe [8]. В спектрах микро-ФЛ ( $T=10 \mathrm{~K})$ для структуры с КТ $\mathrm{CdTe} / \mathrm{Zn}(\mathrm{Mg})(\mathrm{Se}) \mathrm{Te}$ наблюдается набор узких дискретных линий излучения одиночных КТ (левая вставка на рис. 3), при этом их поверхностная плотность может быть оценена как $\sim 10^{10} \mathrm{~cm}^{-2}$.

Таким образом, методом МПЭ были выращены структуры с КТ CdTe в барьерах квазичетверного твердого раствора $\mathrm{ZnMgSeTe}$, реализованного в виде короткопериодных $\mathrm{CP} \mathrm{ZnTe} / \mathrm{MgTe} / \mathrm{MgSe}$, согласованных по периоду решетки с подложкой InAs (001). Определены основные особенности МПЭ таких СР, связанные со спонтанным формированием $\mathrm{ZnSe}$-содержащих слоев на интерфейсах $\mathrm{MgSe} / \mathrm{ZnTe}$ и увеличением доли $\mathrm{MgSe}$ в CP за счет частичного замещения атомов теллура селеном на интерфейсах $\mathrm{MgTe} / \mathrm{MgSe}$. Показано, что снижение потока $\mathrm{Se}$ при росте CP позволяет сократить от нескольких часов до $30 \mathrm{~min}$ паузу после выращивания нижней $\mathrm{CP} \mathrm{ZnTe} / \mathrm{MgTe} / \mathrm{MgSe}$ при закрытом клапане Se-источника, необходимую для нейтрализации влияния Seфона в камере МПЭ на процесс формирования КТ $\mathrm{CdTe} / \mathrm{Zn}(\mathrm{Mg})(\mathrm{Se}) \mathrm{Te}$ методом термоактивации. Спектры микро-ФЛ $(T=10 \mathrm{~K})$ структуры с KT $\mathrm{CdTe} / \mathrm{Zn}(\mathrm{Mg})(\mathrm{Se}) \mathrm{Te}$ демонстрируют набор узких дискретных линий излучения одиночных КТ в спектральном диапазоне $2.0-2.2 \mathrm{eV}$, а оценка поверхностной плотности КТ дает значение $\sim 10^{10} \mathrm{~cm}^{-2}$.

Авторы благодарны А.А. Ситниковой за проведение ПЭМизмерений.

Работа выполнена при поддержке РНФ (проект № 14-22-00107). РДи ПЭМ-измерения выполнены с использованием оборудования Центра коллективного пользования „Материаловедение и диагностика в передовых технологиях“ при частичной финансовой поддержке Министерства образования и науки РФ (соглашение № 14.621.21.0013 от 28.08.2017 г., идентификатор проекта RFMEFI62117X0018).

\section{Список литературы}

[1] Tribu A., Sallen G., Aichele T., André R., Poizat J.-P., Bougerol C., Tatarenko S., Kheng $K$. // Nano Lett. 2008. V. 8. N 12. P. 4326-4329.

[2] Fedorych O., Kruse C., Ruban A., Hommel D., Bacher G., Kümmell T. // Appl. Phys. Lett. 2012. V. 100. N 6. P. 061114.

[3] Couteau C., Moehl S., Tinjod F., Gérard J.M., Kheng K., Mariette H., Gaj J.A., Romestain R., Poizat J.P. // Appl. Phys. Lett. 2004. V. 85. N 25. P. 6251-6253.

Письма в ЖТФ, 2018, том 44, вып. 6 
[4] Sorokin S.V., Sedova I.V., Gronin S.V., Klimko G.V., Belyaev K.G., Rakhlin M.V., Mukhin I.S., Toropov A.A., Ivanov S.V. // J. Cryst. Growth. 2017. V. 477. P. 127130.

[5] Tinjod F., Gilles B., Moehl S., Kheng K., Mariette H. // Appl. Phys. Lett. 2003. V. 82. N 24. P. $4340-4342$.

[6] Kobak J., Rousset J.-G., Rudniewski R., Janik E., Slupinski T., Kossacki P., Golnik A., Pacuski W. // J. Cryst. Growth. 2013. V. 378. P. 274-277.

[7] Wojnar P., Karczewski G., Wojtowicz T., Kossut J. // Acta Phys. Pol. A. 2007. V. 112. N 2. P. 283-288.

[8] Сорокин С.В., Седова И.В., Гронин С.В., Беляев К.Г., Рахлин М.В., Торопов А.А., Мухин И.С., Иванов С.В. // Письма в ЖТФ. 2016. Т. 42. В. 24. C. 64-71.

[9] Grabs P., Richter G., Fiederling R., Becker C.R., Ossau W., Schmidt G., Molenkamp L.W., Weigand W., Umbach E., Sedova I.V., Ivanov S.V. // Appl. Phys. Lett. 2002. V. 80. N 20. P. 3766-3768.

[10] Pacuski W., Kruse C., Figge S., Hommel D. // Appl. Phys. Lett. 2009. V. 94. N 19. P. 191108.

[11] Bonef B., Gérard L., Rouvière J.L., Grenier A., Jouneau P.-H., BelletAmalric E., Mariette H., André R., Bougerol C. // Appl. Phys. Lett. 2015. V. 106. N 5. P. 051904.

[12] Sedova I.V., Sorokin S.V., Sitnikova A.A., Zolotareva R.V., Ivanov S.V., Kop'ev P.S. // Proc. 7th Int. Symp. „Nanostructures: physics and technology“. St. Petersburg, Russia, 1999. P. 547-549.

[13] Rousset J.-G., Kobak J., Janik E., Parlinska-Wojtan M., Slupinski T., Golnik A., Kossacki P., Nawrocki M., Pacuski W. // J. Appl. Phys. 2016. V. 119. N 18. P. 183105. 